\title{
Reply to Dr. Regina and colleagues re: protein-derived acetaminophen-cysteine in supratherapeutic ingestion
}

\author{
Gerald Francis O’Malley ${ }^{1}$
}

Published online: 26 August 2015

(C) American College of Medical Toxicology 2015

\section{Reply to JMT Letter}

We appreciate Dr. Regina's interest in our study; however, we believe she misread and inadvertently mischaracterized the intent and results.

As we stated on the first page of the paper, "The purpose of our study was to determine if protein-derived acetaminophencysteine can be detected after repeated supratherapeutic ingestion in a population of patients in a pain management clinic." Our results support that premise of this prospective study.

We appreciate the time and energy Dr. Regina spent to create the scattergram, but as we explained numerous times to the reviewers and editors during the manuscript acceptance process, we have provided the raw data and do not believe that graphs add anything to the manuscript. It is no surprise that Dr. Regina found a negative correlation when a dozen proteinderived APAP-CYS levels were plotted against self-reported daily APAP dose, as this was never the objective of the study. One additional data point easily could change the correlation coefficient drastically. For instance, if one additional data point in the range of the maximum reported daily dosage and the maximum measured APAP-CYS concentration is added to the data set, the correlation coefficient becomes 0.0025 , and suddenly the weak, statistically insignificant negative correlation becomes a weak, statistically insignificant positive correlation. It is a mistake to try to make any sweeping generalizations on a data set as small as the one described in this manuscript.

As we described in the Discussion section, protein-derived APAP-CYS form as a byproduct of hepatocellular exposure to NAPQI and published data suggest a dose-related creation of protein-derived APAP-CYS in patients that have no demonstrable hepatic injury. Our study was not designed to demonstrate a dose-dependent relationship of protein-derived APAPCYS. Contrary to the concluding sentence of the letter, generation of protein-derived APAP-CYS is clearly related to APAP ingestion in chronic supratherapeutic ingestion, as we demonstrated. We agree with Dr. Regina that much more investigation is necessary to fully understand the contribution of protein-derived APAP-CYS to the development of hepatic injury.

Cordially, Gerald F. O'Malley, DO, FACEP, FAAEM, FACMT, FAACT
Gerald Francis O'Malley

geraldomalley@gmail.com 